Aus der Privatklinik von Dr. J. Voigt und dem lnstitut für anorganische Chemie in Göttingen. (Direktor: Prof. Zsigmondy.)

\section{Biologische Untersuchungen über kolloidales Silber mittels einer neuen Methode zum Nachweis feinster Metallablagerungen in den Organen.)}

\author{
Von J. Voigt.
}

Seit P. Ehrlich auf Grund seiner theoretischen Ucberlegungen und experimentellen Arbeiten - vorzugsweise mit Farbstoffen - als Erster die Bedeutung des distributiven Moments erkannt und gelehrt hat, ist von therapeutisch wichtigen Substanzen nur die Verteilung der Salizylsäure (M. Jakoby) und des Jods (O. L o eb) genauer studiert worden. Es ist dies un so auffallender, als die Berechtigung des Ausspruchs P. Ehrlichs, ,dal3 die Verteilung der Arzneistoffe im Organisınus das Bindeglied zwisehen der ehemisehen Konstitution und der pharmakologiselien Wirkung bilde", von Niemandem ernstlich angefochten werden kann. Das von ihm geforderte Studium der Verteilung bedeutet zugleich das Erfüllen der wichtigsten Vorbedingung für eine wirklich zielbewußte therapeutische Verwendung. Für das kolloidale Silber, das vielfach $z u$ therapeutisehen Zwecken empfohlen wird, ist dieser Forderming noch bei weitem nicht entsproehen; fehlt uns doch anch heute noch die genaue Kenntnis der Verteilung und des Schicksals der Silberhydrosole im tierischen Organismus. Bei der großen, über das kolloidale Silber vorliegenden Literatur ınuß es auffallen, daß es den versehiedenen Untersuchern noch nieht gelungen ist, experimentell feste Grundlagen für seine therapeutisehe Anwendung zu schaffen.

Französische Autoren z. B. haben zicmlich viel ïber dic Frage der Verteilung und des Sichicksals des Siberhydrosols im Organismus gearbeitet. Ihre Beobachtıngen genügen jedoch nicht, um eine Therapic mit kolloidalem Silber wissenschaftlich zu begrïnden; ja es nuß leider festgestellt werden, daß ihre Angaben vi.jfach so wenig genau sind, daB eine Nachprüfung ganz unmöglich ist. Dic weniger zahlreichen deutschen Arbciten, die sich mit dem Studium des Schicksals des Silber. hydrosols in Körper befassen, lassen noch zahlreiche Fragen unbeantwortet.

Auch die sorgfältigste chemische Analyse vermag unterhalb eines gewissen Mininums niehts mehr zu leisten, ganz abgesehen davon, daß die vielen Chloride im Tierkörper einer solchen erhebliche Sehwierigkeiten bereiten. Aus diesem Grunde scheint. auch die sehr mühsame Mikroanalyse hier nicht anwendbar. Auch im besten Falle erhalten wir durch eine quantitative Analyse niemals einen AufsehluB ïber das Verhalten der Zellen, weleher auf deren Funktion resp. Partialfunktion zu sehließen berechtigte. Die quantitativen Untersuchungen finden ihre Ergänzung dureh die mikroskopischen Beobachtungen, deren Bedentung geradc für das Studinm der Verteilung P. Ehrlich nachgewiesen hat. Die gewöhnliche mikroskopisehe Untersuchung vernag zwar Metallniederschläge bis zu einer gewissen geringsten Größe als schwarze oder braune Ablagerungen in den Sehnitten nachzuweisen, aber auch hier ist die Grenze bald erreicht. Wo derartige Niederschläge nieht zu erkennen waren, konnte bisher kein Motall nachgewiesen werden, cbensowenig lie $\beta$ sich entsehciden, ob neben den wahrnehmbaren Ablagerungen nicht noeh feinere beständen, die biologisch wie therapeutiseh von besonderem Interesse sein dürften. Um also nit einiger Aussicht auf Erfolg an das Studium der Verteilung kolloidaler Metalle, wie \%. B. des Silbers, in Organismus gehen zu können, bedurfte man offenbar einer neuen Methode. die uns instandsetzt, möglichst allc. Metallnicdersehläge, insbesondere die bisher nieht sichtbar zu machenden allerfeinsten, im Gewebe nachzuweisen.

Ich bin nun in der Lage, ̈̈ber einc neue Methode zu berichten, die ieh für diese Untersuchungen ausgearbeitet und deren Leistungsfähigkeit ich erprobt habe.

Es gelingt unter Anwendung gewisser Vorsichtsmaßregeln, in genügend feinen Sehnitten nit Hilfe des Paraboloidkondensors dem Auge noch Metallteilehen erkennbar zu machen, die bei der Betrachtung im durehfallenden Iiehte sich

1) Vortrag i. d. Med. Ges. in Göttingen anı 20, X1. 1913. entweder gegenüber der Körnelung des Protoplasmas nicht genügend markieren oder selbst mit stärkster Vergrößerung nicht mehr wahrnehmbar sind. Dies beruht anf der Tatsache, da $B$ feine Partikelehen mit einem anderen Brechungsindex als dem des umgebenden Mediums seitlieh auffallende Strahlen diffus reflektieren und dadureh den Eindruck sclbstleuchtender Gebilde inachen, die sich dann auf dunklem Grund ausgezeichnet abheben. Es ist ohne weiteres klar, da $B$ schon an und für sich ein leuchtender Punkt mit dunkler Ungebung schr viel leichter $\mathrm{zu}$ erkennen ist, als wenn die mmgekehrten Verhältnisse bestehen. Fin weiterer Vorteil dieser Beobachtungsweise liegt noch darin, dal3 die diffuse Reflexion ein Diffraktionsbild, von Zsigmondy u. A. als Beugungsseheibehen bezcichnet, liefert, welehes das lichtbeugende Teilehen an Größe bedeutend übertrifft. Anßer den Niedersehlägen, die immer noch als Ablagerungen von Teilchen zu erkemen sind, kommen auch solehe Bilder vor, die dureh Adsorption allerfeinster, ultramikroskopiseher Metallteilehen zu erklären sind; es treten hier keine Beugungsseheibehen mehr auf, sondern es geht ein feiner Liehtsehein von der betreffenden Membran aus.

Während es bei der ultramikroskopischen Untersuchung von Metallhydrosolen nieht angängig ist, von der Farbe des Beugungsseheibehens auf die Größe des lichtbengenden Teilehens zu schließen, so seheint nach meinen Beobachtungen bei meiner Methode ein derartiges Vorgehen bedingt zulässig, wenn es sich um Sehnitte handelt, in denen Silberablagerungen vorhanden sind. (Ob die gleich zu besehreibenden Verhältnisse aneh für andere Metallhydrosole zutreffen, kann ich nach dem mir bis jetzt vorliegenden Material noeh nicht entsehciden.) Die Farbe des dureh Niederschläge eines Silberhydrosols abgebeugten Lichtes ist sehr verschieden; sie durchläuft alle Nuancen von dunklen Goldrot, das sich z. B. an den Fmboli aus Silberteilchen findet, über Goldgelb zum Silberweiß, um bei den erwähnten Fällen von Imprägnierung zu einem hellstrahlenden Himmelblau zu gelangen.

Nach meiner Frfahrung sind für die Technik der Dunkelfelduntersuehung von Schnitten zum Studium der Verteilung kolloidaler Metalle in den Organen besonders folgende Punkte von Bedeutung:

Das Protoplasma besitzt selber ein gewisses Lichtbeugungsvermögen, das sich bei Verwendung einer sehr starken Lichtquclle störend geltend macht, da dic einzclnen leuchtenden Yetallteilehen sich auf dem hellschintmernden Protoplasılı nicht genügend dentlich abheben können. Dic besten Bilder erhält Inan bei klarcm Himmel ohne dircktes Sonnenlicht, sonst genïgt auch cine 50 kerzige Osramlanpe, wenn man die Vorsicht gebraucht, eine matticrte Blauscheibe davor zu stellen. Uin bei dicsem verhältnismäßig schwachen Iicht untersuchon zu können, muß man nach Möglichkeit alles Nebcnlicht von Auge ferniralten.

Dess Weiteren ist für diesc Lntersuchungen in Dunkelfeld die denkbar peinlichste R(-inheit jełlen Zubehörs von größter Becleutung. Deckglüser und Objekttrïgcr müssen in konzentricrter Salpetersuiure ausgekocht und in Alkohol aufbewahrt werden, alle Reagentien sind nur frisch filtricrt zu verwenden. Der Kanadabalsam 1 nuß wenigstens von ZCit zu \%cit im Dunkelfeld daraufhin untersucht werden, ob er kcinc: nonnenswerten Verunreinignngen enthält. In den neisten Präparat'’n werden sich trotzdem einzelne lichtbcugende Punkte finden, dic nicht den gesuchten Metallteilehen entsprechen. Um sich da vor Täuschungen zu bewahren, ist es ratsam, cine gantze Reihe von Sichitten zu untersuchen, die mit verschicdenen Reagentien behandelt worden sind. Einige Niederschlägc versehwinden in verdünnter Salzsäure, andere in Kalilauge, in manchen Fällen ist ein intensives Behandeln mit Xylol \%u empfehlell. Nur dicjenigen lichtbeugenden Teilehen, welche allein auf Zusetren eles spezifisehen Lösungsinittels - fïr nıeine Versuehe mit Silber $1 \%$ ige Yyankalilossung - versehwinden, kann man nit Sicherheit als dic gesuchten Ablagerungen ansprechell. Die sich hier und da in den Schnitten findenden Verunreinigungen dureh Staubteilchen sind uncist schon dureh dic Inkonstan\% ihrer Lage im Gewcbe eharakterisicrt. Es muß aber noch auf cino Fehlerquelle hingewiesen werden, die nieht gut auszuschalten ist. Zuwcilen komınt es vor, daß durch die Klinge des Mikrotons einzelne Metallteilchen im Gewrbe woitcrge. schoben werden -- es ist das am häufigsten bei gröberen Ablagerungen der Fall -- - und dann falsche: Bilder crzcugen. Es genügt aber wohl stets, an dicse Möglichkeit zu denken, um derartigen Täuselıungen zu ent. gchen.

Ungefärbte Sehnitte sind im ganzen für die Dunbelfeldunter. suchung vorzuziehen, doch nöchte ich empfehlen, zum Vergleich auch nit Hä̈natoxylin diskret gefärbte Präparate heranznziehon. 1)as Be. trachten letyterer, abwechsclnd inı $\mathrm{Hell}$. und D)unkelfeld, crnöglicht 
cin genanes Lokalisieren der einzelnen Niedersebläge im Gewebe. $\Lambda$ ls cin wcitercs Hilfsmittcl dafür hat sich mir folgendes Verfabren gut bewährt. Alle in durehfallenden Licht erkennbaren Niedersehläge werden zunächst nit Hilfe des $\Lambda$ b b éc. Winkelsehen Zeichenapparates gezeichnet. Nach Herstellen des Dnnkelfeldes muß durch Hinschalten eines passenden Rauehglases dic Hclligkeit der Zeichenfläehe vermindert werden; ınan kann danı dic eben beobachteten Niedersehläge mit den im J)unkelfeld leuchtenden Punkten identifizieren und markieren, a u Berden aber noch erkennbar werdende dazu zeichnen. Sehr viel eleganter ist natiurlich der Ausweg, das nikroskopische Bild sowohl im Hellfeld wic im Dunkelfeld zu photographieren. Dazu muß ich jedoch benerken, daß die Mikrophotographie im Dunkelfeld nicht imnıer tin 80 gutes Bild liefert, wie der beim Betrachten erhaltene Eindruck erwarten lie.B. Abgeschen davon, daß eine Randschärfe selbst unit den bestel Systemen nicht zu erzielen ist - es liegt das in der Konstruktion cles Kondensors begriindet —, schaltet der Beobachter störende Reflexe ete. mehr oder weniger unbewußt ans, die auf der photographisehen Platte eine Lnklarheit des IBildes bedingen. Un möglichst gute Aufnahmen zll erhalten, empfehle ich, das Präparat nit nüglichst sehwacher V(rgrößcrung (:inzustcllen und durch Ausziehen des Kamerabalges mit der Platte so weit \%uriiekzugehen, $d a ß$ nur der nittlere'Teil des Gesichtsfeldes fiir dic: Aufnahme in Frage kommt. I)ie so erzielte Vergrößcrung entspriclit. der eines bedeutend stärkeren Systenis, und das Bild ist von Verzeichnungen fast vollständig frei. Für solehe Aufnahmen ist eine sehr lang: Expositionszeit nötig; sie sehwankte bei Anwendung cines Auerbrenners als Liehtquelle bei meinen Versuehen zwisehen 1 Stunde und $4 \frac{1}{2}$ Stunden.

Der Gebrauch einer Sternblende unter Verwendung des gewöhn. lichın Kondensors genügt für Uebersichtsbilder; zmın Stıdium feinster Metallniederschläge bedarf nan aber unbedingt eines guten Para. boloidkoudensors, der anf eine bestimmtc J)icke (ler Objektträger cingestellt ist, die dann selbstverständlich aussehlicßlich gebraucht werden solltell. Sodann ist zu übcrlegen, ob nicht ein Vermindern der Apertur durch das Aufklcben einer Papierseheibe auf die Unterseite des Kondensurs oder $\mathrm{r}$ etwas Aehnliches für den Nachweis stark lieht. beugender Teilchen in Gewebe von Vorteil wäre.

Die Leistmmgsfähigkeit meiner Methode dureh Messung der liehtbeugenden Teilehen etwa zu bestimmen, gelingt nicht, doch mögen folgende Tatsachen cine Vorstellung davon geben:

Die Membrana propria gewisser Harnkanälchen in bestimmten Präparaten, dic in der Durchsieht keinerlei Silberniederschläge erkennen ließen, strahlten im Dunkelfeld in hellblauenn Lichte, das anf Zissetzen von Zyankalilösung, aber $n \mathrm{nr}$ daraufhin, sofort verschwand. Bei einem mittelgroßen Kaninchen, das in Forn einer intravenösen Injektion $0,00125 \mathrm{~g}$ kolloidales Goll erhalten hatte, konnte man in der Leber im Dunkelfeld die Goldnicdersehläge an den typisehen Stellen nit aller Sicherheit nachweisen, --- sic wurden chenfalls nur von Zyankalilösung zun Versehwinden gebracht - die im Hellfeld selbst mit Hilfe der Imınersion nicht sicher zil bestinunen waren.

Die Tatsache, daß es nit der neuen Methode gelingt, so feine Metallablagerungen in Gewebe zu erkennen, eröffnet neue Perspektiven, die zu verfolgen nicht nur vom rein pharmakologisehen, sondern anch vom pliysiologisehen, experimentell pathologiselien und experimentell therapeutisehen Gesichtspunkte aus lolınen dürfte.

Meine Versuche sind, wie ich bereits erwähnte, im wesentliehen mit kolloidalen Silber angestellt worden; icl habe jedoch Anhaltspunkte dafür, daß sich das Studium anderer Metallsole und wahrselieinlich auch anderer Kolloide, sowie möglicherweise aucl anderer Netallverbindungen mittels des neuen Verfalirens als fruchtbar erweisen wird.

Vom pliarmakologischen Standpunkte aus ist das Studium der einzelnen Metallhydrosole nicht nur mit Rücksicht auf ihre Verteilung in Organismus interessant, es gibt aluch einen Hinwejs auf gewisse therapeutische Möglichkeiten. Außerdem wird es aber auch mit Sicherheit mittels dieses Verfahrens gelingen, die verschiedenen Silberhydrosole z. B. untereinander auf ihren Wert zu vergleichen, was vielleicht auch für die Arzncimittelprüfung und-bewertung in Frage käme.

Aus dem normalen Verhalten der kolloidalen Metalle im Organismus dürften konstante Befunde vielleicht Rüekschlüsse auf die Physiolog ic cinzelner Organe und Zellgruppen crlauben. Gelingt es uns, eine Verwandtsehaft des Silberhydrosols oder eines anderen kolloidalen Wetalls z. B. zu den Endothelien nachzuweisen, so dürften wir beispielsweise Aufsehlïsse über die normalen I.ymphbahnen durch besondere Färbung der Lynıphapillaren erwarten. Die Neigung, in bestimmten Zellgebilden, wie in den Sternzellen der Leber oder in bestimmten
Nierenzellen, sich abzulagern, weist uns auf besondere physiolog is che Verhältnisse hin. Kennen wir aber diese, so können wir sie auch durch die verschiedenartigsten Eingriffe stören, sodaß die Verteilung der kolloidalen Metalle eine Veränderung erleidet. So können wir hoffen, sehr feine Störungen in der Funktion der Organe oder einzelner Zellgruppen zu erkennen, die auf andere Weise nicht festzustellen sind, denn die Störung einer Funktion braucht noch keineswegs grob histologiseh erkennbare Veränderungen zu bedingen.

Vom praktisehen Standpunkte aus dürften sich experimentell-therapeutische Versuche lohnen. Es wäre z. B. die Verteilung bei infizicrten Tieren zu studieren. Man könnte einen Zusammenhang zwisehen der Art der Verteilung eines Silberhydrosols oder anderer kolloidaler Metalle und der Wirksamkeit einerseits, und ciner veränderten Verteilung im pathologisehen Organismus anderseits, wie sie von Loeb bereits für das Jod nachgewiesen wurde, aufsuehen. is würde das nicht nur theoretisch Interessantes liefern, sondern auch für die therapeutische 'Teehnik unter Umständen von Bedeutung scin. Bei Trypanosomeninfektion wäre aueh eine eventuelle Anhäufung der Metallteilchen in lebenden Infektionserreger z,1l studieren. Seit längerer Zeit beschäftigt mich die Frage des Verhaltens der kolloidalen Metalle dem Karzinon gegeniiber. Insbesondere wird sich bei den experimentellen Mäuse- und Rattentumoren aus dem Studium der Verteilung mittels des beschriebenen Verfahrens die Frage diskutieren lassen, ob die Metalle lediglich als Kapillargifte wirken, oder ob auch eine Wirkung auf die Tumorzellen selbst anzunchmen ist. Der Nachweis ihrer Ablagerung im Tumorgewebe würde auch die bereits theroretiseh empfohlene Kombination der Strahlen. therapie von Karzinomen mit Injektionen von kolloidalem Metall bereshtigt erseheinen lassen.

Ich darf vielleicht «um Sehlusse noch darauf hinweisen, da $\beta$ aueh vom gerichtlich-medizinisehen, insbesondere vom toxikologisehen Standpunkt aus die nene Methode sieh als fruchtbar erweisen dürfte. 